\title{
Distribution and antimicrobial activity of Thai marine actinomycetes
}

\author{
Wongsakorn Phongsopitanun ${ }^{1,2}$, Khanit Suwanborirux ${ }^{3}$, Somboon Tanasupawat ${ }^{1^{*}}$ \\ ${ }^{1}$ Department of Biochemistry and Microbiology, Faculty of Pharmaceutical Sciences, Chulalongkorn University, Bangkok, Thailand. \\ ${ }^{2}$ Department of Biology, Faculty of Science, Ramkamhaeng University, Bangkok, Thailand. \\ ${ }^{3}$ Department of Pharmacognosy and Pharmaceutical Botany, Faculty of Pharmaceutical Sciences, Chulalongkorn University, Bangkok, Thailand.
}

\begin{tabular}{l}
\hline ARTICLE INFO \\
\hline Received on: $18 / 11 / 2018$ \\
Accepted on: $17 / 12 / 2018$ \\
Available online: $28 / 02 / 2019$
\end{tabular}

Key words:

Antibacterial activity, marine actinomycetes, sediment, marine sponges.

\begin{abstract}
A total of 41 actinomycetes were isolated from marine samples collected in Thailand. On the basis of morphology, chemotaxonomy, and 16S rRNA gene sequence analysis, they were identified as Salinispora (13 isolates), Micromonospora (11 isolates), Nocardia (1 isolate), Verrucosispora (2 isolates), and Streptomyces (14 isolates). The antimicrobial activity screening revealed that two Micromonospora isolates, 12 Salinispora isolates and 10 Streptomyces isolates showed activity against Staphylococcus aureus ATCC 25923, Kocuria rhizophila ATCC 9341, Bacillus subtilis ATCC 6633, Escherichia coli NIHJ KC213, Candida albicans KF1, and Mucor racemosus IFO 4581. Based on this study, the production media and strains were the main factors that influenced the antimicrobial activity.
\end{abstract}

\section{INTRODUCTION}

Actinomycetes are Gram-positive filamentous bacteria having high mol $\%$ of the base guanine plus cytosine content in their genomes (Stackebrandt et al., 1997). They have been well known as the valuable economically importance microorganisms for a long time because of their ability to produce a large number of bioactive secondary metabolites (Berdy, 2005). In general, actinomycetes are widely distributed in terrestrial habitats, mainly in soils, organic materials, and plant materials (Goodfellow et al., 1988; Qin et al., 2012). Since they have been isolated from these habitats for a century, numerous redundant isolates were obtained. Three-quarters of the planet earth covers with the oceans which contain a huge biological diversity. Since the discovery of the true obligate marine actinomycetes in the genus Salinispora in the last decade, the ocean has been accepted for the existence of the actinomycetes. Moreover, the distribution of actinomycetes in the marine environment are largely unexplored (Bister et al., 2004; Jensen et al., 2007; Kwon et al., 2006). The aim of this study is

\footnotetext{
*Corresponding Author

Somboon Tanasupawat, Department of Biochemistry and Microbiology, Faculty of Pharmaceutical Sciences, Chulalongkorn University, Bangkok, Thailand.E-mail: Somboon.T@ chula.ac.th
}

to isolate, identify and screen for antimicrobial activities of the marine actinomycetes isolated from sand, sediments and marine sponges collected in Thailand.

\section{MATERIALS AND METHODS}

\section{Samples collection and isolation methods}

The marine samples, including sand, sediments and marine sponges, were collected from Chumphon (Chumphon beach and Koh Khai), Chonburi (the nature education center for mangrove conservation and ecotourism, and Bangsaen beach), Phuket (Phanwa beach), Trang (Koh Rok Nork, Koh Rok Nai, and Koh Mah) and Krabi Province (mangrove forest) using scuba diving gears. They were isolated using the standard dilutionplating method. One gram of samples was suspended in $9 \mathrm{ml}$ of sterile natural seawater to make 10-fold dilution series to $10^{-4}$. Each diluted suspension $(0.1 \mathrm{ml}$ ) was spread on M1, M2 (Zhang et al., 2006) and seawater-proline media (Inahashi et al., 2011; modified with seawater) supplemented with nalidixic acid $25 \mu \mathrm{g} \mathrm{ml}^{-1}$ and cycloheximide $50 \mu \mathrm{g} \mathrm{ml}^{-1}$. The plates were incubated at $28^{\circ} \mathrm{C}$ for 30 days. The colonies of marine actinomycetes were observed using a light microscope and were transferred to ISP2 agar plates (Shirling and Gottlieb, 1966). The purified cultures were maintained on ISP2 medium at $4^{\circ} \mathrm{C}$. All isolates were preserved using freeze-drying and freezing at $-80^{\circ} \mathrm{C}$ in $15 \%(\mathrm{v} / \mathrm{v})$ glycerol solution. 


\section{Identification methods}

\section{Phenotypic, chemotaxonomic, and genotypic characterization}

The marine actinomycete isolates were identified using the phenotypic and genotypic characteristics. Morphological characteristics of the isolates were observed on the culture grown on ISP2 medium at $28^{\circ} \mathrm{C}$ for 14 days. Cultural characteristics were determined using 14-day cultures grown at $28^{\circ} \mathrm{C}$ on yeast extract-malt extract medium (ISP2 medium). The isomers of diaminopimelic acid were analyzed using the standard TLC method (Staneck and Robert, 1974).

The genomic DNA for 16S rRNA gene amplification was extracted from the cells using the method as described by Tamaoka (1994). The amplification of 16S rRNA gene was carried out using two primers 20F (5'-GAGTTTGATCCTGGCTCAG-3', positions 9-27) and 1500R (5'-GTTACCTTGTTACGACTT-3', positions 1492-1509). The PCR (Polymerase chain reaction) mixture (final volume $100 \mu \mathrm{l}$ ) contained $4 \mu \mathrm{l}$ each of primers $(10$ $\mathrm{pmol} / \mu \mathrm{l}), 2 \mu \mathrm{l}$ of dNTP $(10 \mathrm{mM}), 10 \mu \mathrm{l}$ of 10x Taq buffer, $8 \mu \mathrm{l}$ of $\mathrm{MgCl}(25 \mathrm{mM}), 0.5 \mu \mathrm{l}$ of Taq DNA polymerase, $61.5 \mu \mathrm{l}$ of $\mathrm{dH}_{2} \mathrm{O}$, and $10 \mu \mathrm{l}$ of template DNA. The amplification was performed with an initial denaturation at $94^{\circ} \mathrm{C}$ for 3 minutes, followed by 30 cycles with denaturation at $94^{\circ} \mathrm{C}$ for 1 minute, annealing at $50^{\circ} \mathrm{C}$ for 1 minute and extension at $72^{\circ} \mathrm{C}$ for 2 minutes, followed by the last step at $72^{\circ} \mathrm{C}$ for 3 minutes (Suriyachadkun et al., 2009). The PCR product was purified using the PCR purification kit (Gene aid). The sequencing of nucleotides was performed using universal primers, 27F (5'-AGAGTTTGATCMTGGCTCAG-3') and 800R (5'-TACCAGGGTATCTAATCC-3') (Lane, 1991) (Macrogen; Seoul, Korea).

\section{Antimicrobial activities}

The culture broth library was prepared by using four different production media including 301 medium, 54 medium (2 $\mathrm{g}$ soluble starch, $0.5 \mathrm{~g}$ glycerol, $1 \mathrm{~g}$ defatted wheat germ, $0.3 \mathrm{~g}$ meat extract, $0.3 \mathrm{~g}$ yeast extract, $0.3 \mathrm{~g} \mathrm{CaCO}_{3}, 500 \mathrm{ml}$ tap water, $500 \mathrm{ml}$ artificial seawater, $\mathrm{pH}$ 7.4-7.8), 51 medium, and yeast-dextrose (YD) broth (Sripreechasak et al., 2013; 2014). The inoculum of each strain was cultured in yeast extract-dextrose broth for 4-7 days. Then, $0.1 \mathrm{ml}$ of the culture was transferred to $10 \mathrm{ml}$ of the screening production media and incubated in a shaking condition at $180 \mathrm{rpm}$ at $30^{\circ} \mathrm{C}$ for $7-14$ days. After incubation, $10 \mathrm{ml}$ of $95 \%$ ethanol were added into the culture broth and shaked at $180 \mathrm{rpm}$ for 2 hours. The extract solution was centrifuged at 3,400 rpm for 15 minutes and preserved at $-20{ }^{\circ} \mathrm{C}$. The production media without the culture was used as the negative control.

The screening of antimicrobial activities were performed using agar disc diffusion method (Qin et al., 2009). Each of paper disc $(8 \mathrm{~mm})$ was soaked into the extract solution and air-dried. After drying, the discs were put onto the surface of the agar plate containing a tested microorganism and cooled at $4^{\circ} \mathrm{C}$ for 30 minutes before incubation. The bacterial plates were incubated at $37^{\circ} \mathrm{C}$ for 24 hours while yeast and filamentous fungus were incubated at $30^{\circ} \mathrm{C}$ for 48 hours. The inhibition zones $(\mathrm{mm})$ were measured using a Vernier caliper. Three bacteria, Bacillus subtilis ATCC 6633, Staphylococcus aureus ATCC 25923, Escherichia coli NIHJ KB213, one yeast, Candida albicans KF1, and one filamentous fungi Mucor racemosus IFO $4581^{\mathrm{T}}$ were used as the tested microorganisms.

\section{RESULTS AND DISCUSSION}

\section{Identification}

On the basis of 16S rRNA gene sequence analysis and morphological characteristics, the actinomycete isolates were belonged to the family Micromonosporaceae, Nocardiaceae and Streptomycetaceae (Table 1). They were identified as Salinispora (13 isolates), Micromonospora (11 isolates), Nocardia (1 isolate), Verrucosispora (2 isolates), and Streptomyces (14 isolates).

Group I, the members of this group exhibited a monomeric spore on the substrate mycelia but lacked aerial mycelia. They contained meso-diaminopimelic acids. They were divided into three genera based on the key morphological and chemotaxonomic characteristics and16S rRNA gene sequence analysis including phylogenetic tree relationship.

Group IA comprised 13 isolates (KRN2-1, SPM3-5, SPM3-1, SPM3-3, SPM3-6, SPM3-7, SPM9-1, SPM9-2, SRK11, SRK1-2, SRK1-3, SRK2-1 and SRK2-3) (Table 1). All strains required sea water for the growth. The $16 \mathrm{~S}$ rRNA gene analysis revealed that these isolates showed the highest similarity with Salinispora arenicola $\mathrm{CNH}-643^{\mathrm{T}}$ (100\% similarity). Therefore, they were identified as S. arenicola (Maldonado et al., 2005).

Group IB consisted of 11 isolates (BS-003, BS-007, CH7-4m, CPB1-11, CPB1-21, KK1-10, PW-001, PW-002, PW-004, PW-006 and PWB-012) (Table 1). Almost all isolates showed orange to brown substrate mycelia which changed into a dark brown or black color when cultured more than 10 days. Based on BLASTn and phylogenetic analyses, these strains showed the highest 16S rRNA gene sequence similarities with type strains of the members of the genus Micromonospora (Kawamoto, 1989).

Group IC Verrucosispora comprised two isolates including KK2-1 and SPM3-8 (Table 1). The representative isolate KK2-1 produced hairy spores which born singly on the substrate mycelia. BLASTn analysis of 16S rRNA gene sequences revealed that the isolates KK2-1 and SPM3-8 were the closest similar to Verrucosispora gifhornensis DSM $44337^{\mathrm{T}}(99.7 \%)$ and Verrucosispora sediminis MS426 $^{\mathrm{T}}(99.5 \%)$, respectively. On the basis of $16 \mathrm{~S}$ rRNA gene sequence, they were identified as Verrucosispora (Rheims et al., 1998).

Group II consisted of one isolate, PWB-002. This isolate showed the fragmentation on the substrate mycelia and contained meso-diaminopimelic acid in cell-wall peptidoglycan. It showed the phylogenetic relationship within the genus Nocardia. Based on the fragmentation of substrate mycelia and 16S rRNA gene sequence analysis, this isolate was identified as Nocardia (Kageyama et al., 2004).

Group III, 14 isolates including SRN1-2, LKB14, KK5-10, KRN1-1, PWB-011, BM2-4, CPB1-13, LKB1-6, PWB-010, PWB-016, SRN1-1, LKB1-5, LKB1-7 and LKB1-11 (Table 1). They produced extensively branch aerial and substrate mycelia. Almost all isolates produced spiral spore chains, while long straight spore chains were occasionally observed. The chemotaxonomic analysis revealed that all of these isolates contained LL-diaminopimelic acids. In addition, the 16S rRNA gene sequence analysis (ranged from $98.2 \%$ to $99.9 \%$ similarity), these isolates shared the clade within the genus Streptomyces (Mao et al., 2007). 
Table 1. Isolate number, sources of isolation, length of nucleotide sequence, accession no., 16S rRNA gene sequence similarity (\%) and nearest relatives.

\begin{tabular}{|c|c|c|c|c|c|}
\hline Isolate no. & Source & Base pair (nt) & Accession no. & Similarity (\%) & Nearest relatives \\
\hline 1.KRN2-1 & Sediment ${ }^{6}$ & 650 & LC158533 & 100 & S. arenicola $\mathrm{CNH}-643^{\mathrm{T}}$ \\
\hline 2.SRK2-1 & Sponge $^{7}$ & 660 & LC158534 & 100 & S. arenicola $\mathrm{CNH}-643^{\mathrm{T}}$ \\
\hline 3.SRK1-3 & Sponge $^{7}$ & 640 & LC158535 & 100 & S. arenicola $\mathrm{CNH}-643^{\mathrm{T}}$ \\
\hline 4.SPM9-1 & Sponge $^{8}$ & 679 & LC158536 & 100 & S. arenicola $\mathrm{CNH}-643^{\mathrm{T}}$ \\
\hline 5.SPM3-7 & Sponge $^{8}$ & 639 & LC158537 & 100 & S. arenicola $\mathrm{CNH}-643^{\mathrm{T}}$ \\
\hline 6.SPM9-2 & Sponge $^{8}$ & 650 & LC158538 & 100 & S. arenicola $\mathrm{CNH}-643^{\mathrm{T}}$ \\
\hline 7.SPM3-6 & Sponge $^{8}$ & 649 & LC158539 & 100 & S. arenicola $\mathrm{CNH}-643^{\mathrm{T}}$ \\
\hline 8.SPM3-3 & Sponge $^{8}$ & 609 & LC158540 & 100 & S. arenicola $\mathrm{CNH}-643^{\mathrm{T}}$ \\
\hline 9.SPM3-1 & Sponge $^{8}$ & 630 & LC158541 & 100 & S. arenicola $\mathrm{CNH}-643^{\mathrm{T}}$ \\
\hline 10.SRK1-2 & Sponge $^{8}$ & 609 & LC158542 & 100 & S. arenicola $\mathrm{CNH}-643^{\mathrm{T}}$ \\
\hline 11.SRK2-3 & Sponge $^{8}$ & 600 & LC158543 & 100 & S. arenicola $\mathrm{CNH}-643^{\mathrm{T}}$ \\
\hline 12.SRK1-1 & Sponge $^{8}$ & 550 & LC158544 & 100 & S. arenicola $\mathrm{CNH}-643^{\mathrm{T}}$ \\
\hline 13.SPM 3-5 & Sponge $^{8}$ & 640 & LC158545 & 100 & S. arenicola $\mathrm{CNH}-643^{\mathrm{T}}$ \\
\hline 14.PW-001 & Sediment ${ }^{1}$ & 560 & LC158516 & 100 & M. aurantiaca ATCC $27029^{\mathrm{T}}$ \\
\hline 15.BS-007 & Sand $^{2}$ & 600 & LC158517 & 100 & M. aurantiaca ATCC $27029^{\mathrm{T}}$ \\
\hline 16.PW-002 & Sediment $^{1}$ & 580 & LC158518 & 99.66 & M. aurantiaca ATCC $27029^{\mathrm{T}}$ \\
\hline 17.BS-003 & Sand $^{2}$ & 530 & LC158520 & 99.81 & M. chalcea DSM $43026^{\mathrm{T}}$ \\
\hline 18.CH7-4m & Mangrove sediment ${ }^{3}$ & 559 & LC158521 & 99.46 & M. chersina ATCC $27029^{\mathrm{T}}$ \\
\hline 19.KK1-10 & Sediment ${ }^{4}$ & 579 & LC158528 & 100 & M. marina JSM $1-1^{\mathrm{T}}$ \\
\hline 20.CPB1-11 & Sediment $^{5}$ & 549 & LC158529 & 100 & M. marina JSM $1-1^{\mathrm{T}}$ \\
\hline 21.CPB1-21 & Sediment $^{5}$ & 600 & LC158530 & 100 & M. marina JSM $1-1^{\mathrm{T}}$ \\
\hline 22.PW-004 & Sediment ${ }^{1}$ & 599 & LC158531 & 98.3 & M. olivasterospora DSM $43868^{\mathrm{T}}$ \\
\hline 23.PWB-012 & Sand $^{1}$ & 569 & LC158510 & 99.82 & M. tulbaghiae $\mathrm{TVU}^{\mathrm{T}}$ \\
\hline 24.PW-006 & Sediment ${ }^{1}$ & 569 & LC158532 & 100 & M. tulbaghiae TVU1 ${ }^{\mathrm{T}}$ \\
\hline 25.KK2-1 & Sediment ${ }^{4}$ & 630 & LC158547 & 99.68 & V. gifhornensis DSM $44337^{\mathrm{T}}$ \\
\hline 26.SPM3-8 & Sponge $^{8}$ & 609 & LC158548 & 99.51 & V. sediminis $\mathrm{MS} 46^{\mathrm{T}}$ \\
\hline 27.PWB-002 & Sand $^{1}$ & 509 & LC158550 & 99.21 & N. testacea $\mathrm{NBRC} 100365^{\mathrm{T}}$ \\
\hline 28.SRN1-2 & Sponge $^{7}$ & 630 & LC158551 & 98.25 & S. gulbargensis DAS131 ${ }^{\mathrm{T}}$ \\
\hline 29.LKB1-4 & Mangrove sediment ${ }^{9}$ & 470 & LC158552 & 99.57 & S. coelicoflavus NBRC $15399^{\mathrm{T}}$ \\
\hline 30.KK5-10 & Sediment ${ }^{4}$ & 589 & LC158554 & 99.46 & S. coelicoflavus NBRC $15399^{\mathrm{T}}$ \\
\hline 31.KRN1-1 & Sediment ${ }^{6}$ & 610 & LC158556 & 99.18 & S. platensis JCM $4662^{\mathrm{T}}$ \\
\hline 32.PWB-011 & Sand $^{1}$ & 410 & LC158558 & 99.02 & S. olivaceoviridis $\mathrm{NBRC} 13066^{\mathrm{T}}$ \\
\hline 33.BM2-4 & Sediment $^{2}$ & 535 & LC158560 & 99.07 & S. radiopugnans $\mathrm{R} 97^{\mathrm{T}}$ \\
\hline 34.CPB1-13 & Sediment $^{5}$ & 600 & LC158561 & 99.17 & S. radiopugnans $\mathrm{R} 97^{\mathrm{T}}$ \\
\hline 35.LKB1-6 & Mangrove sediment ${ }^{9}$ & 560 & LC158562 & 97.68 & S. rimosus DSM $41429^{\mathrm{T}}$ \\
\hline 36.SRN1-1 & Sponge $^{6}$ & 630 & LC158563 & 98.25 & S. gulbargensis DAS131 ${ }^{\mathrm{T}}$ \\
\hline 37.LKB1-5 & Mangrove sediment ${ }^{9}$ & 619 & LC158564 & 99.84 & S. sanyensis $219820^{\mathrm{T}}$ \\
\hline 38.LKB1-7 & Mangrove sediment ${ }^{9}$ & 509 & LC158565 & 99.8 & S. sanyensis $219820^{\mathrm{T}}$ \\
\hline 39.PWB-010 & Sand $^{1}$ & 597 & LC158566 & 98.15 & S. somaliensis DSM $40738^{\mathrm{T}}$ \\
\hline 40.PWB-016 & Sand $^{1}$ & 589 & LC158567 & 98.64 & S. somaliensis DSM $40738^{\mathrm{T}}$ \\
\hline 41.LKB1-11 & Mangrove sediment ${ }^{9}$ & 630 & LC158568 & 99.84 & S. tritolerans DAS $165^{\mathrm{T}}$ \\
\hline
\end{tabular}

'Phanwa beach, Phuket; ${ }^{2}$ Bangsaen beach, Chonburi; ${ }^{3}$ The nature education center for mangrove conservation and ecotourism, Chonburi; ${ }^{4}$ Koh Khai, Chumphon; ${ }^{5}$ Chumphon beach, Chumphon; ${ }^{6} \mathrm{Koh}$ Rok Nai, Trang; ${ }^{7}$ Koh Rok Nork, Trang; ${ }^{8}$ Koh Mah, Trang; and ${ }^{9}$ Mangrove forest, Krabi.

\section{Antimicrobial activities}

Fourteen isolates of the family Micromonosporaceae showed antimicrobial activities against tested microorganisms. Twelve isolates from genus Salinispora including the isolates SRK2-1, SRK1-3, SPM9-1, SPM3-7, SPM9-2, SPM3-6, SPM3-1, SRK1-2, SRK2-3, SRK1-1 and SPM3-5 showed antimicrobial activities against $S$. aureus ATCC 25923, Kocuria rhizophila ATCC 9341 and B. subtilis ATCC 6633, while one isolate, KRN21 showed activities against $S$. aureus ATCC 25923 Tables 2 and 3. All of these isolates were identified as S. arenicola.

Two isolates of the genus Micromonospora including the isolate $\mathrm{CH} 7-4 \mathrm{~m}$ and PW-004 showed antimicrobial activities 
Table 2. Antimicrobial activities of Salinispora, Micromonospora and Streptomyces isolates when cultured in 301 and YD media.

\begin{tabular}{|c|c|c|c|c|c|c|c|c|c|c|c|c|}
\hline \multirow{3}{*}{ Isolates } & \multicolumn{12}{|c|}{ Inhibition zone (mm) } \\
\hline & \multicolumn{6}{|c|}{ Cultured in 301 medium } & \multicolumn{6}{|c|}{ Cultured in YD medium } \\
\hline & $S$. & $K$ & $B$ & $E$ & $C$ & $M$ & $S$ & $K$ & $B$ & $E$ & $C$ & $M$ \\
\hline \multicolumn{13}{|l|}{ Salinispora } \\
\hline SPM3-7 & - & - & - & - & - & - & - & 9.5 & - & - & - & - \\
\hline SPM3-1 & - & - & - & - & - & - & - & 8.4 & - & - & - & - \\
\hline SRK1-2 & - & - & - & - & - & - & - & 19.9 & - & - & - & - \\
\hline \multicolumn{13}{|c|}{ Micromonospora } \\
\hline $\mathrm{CH} 7-4 \mathrm{~m}$ & 9.1 & 16.7 & 22.7 & - & - & - & 15.7 & 13.3 & 14.8 & - & - & - \\
\hline PW-004 & - & - & - & - & - & - & - & - & 13.4 & - & - & - \\
\hline \multicolumn{13}{|l|}{ Streptomyces } \\
\hline BM2-4 & 15.5 & 17.5 & 11.0 & - & - & - & 17.0 & 17.6 & 12.5 & - & - & - \\
\hline CPB1-13 & 14.4 & 14.9 & 9.8 & - & - & - & 22.9 & 17.2 & 11.6 & - & - & - \\
\hline PWB-010 & - & 9.6 & 8.2 & - & - & - & - & 10.7 & 10.8 & 11.3 & - & - \\
\hline PWB-016 & - & 11.4 & 8.3 & - & - & - & & 12.0 & 9.0 & & & \\
\hline
\end{tabular}

Table 3. Antimicrobial activities of Salinispora, Micromonospora and Streptomyces isolates when cultured in 54 and 51 media.

\begin{tabular}{|c|c|c|c|c|c|c|c|c|c|c|c|c|}
\hline \multirow{3}{*}{ Isolates } & \multicolumn{12}{|c|}{ Inhibition zone (mm) } \\
\hline & \multicolumn{6}{|c|}{ Cultured in 54 medium } & \multicolumn{6}{|c|}{ Cultured in 51 medium } \\
\hline & $S$ & $K$ & $B$ & $E$ & C & $M$ & $S$ & $K$ & $B$ & $E$ & $C$ & $M$ \\
\hline \multicolumn{13}{|l|}{ Salinispora } \\
\hline KRN2-1 & - & - & - & - & - & - & 11.7 & - & - & - & - & - \\
\hline SRK2-1 & 14.5 & - & - & - & - & - & 15.6 & 24.8 & 8.9 & - & - & - \\
\hline SRK1-3 & 8.7 & 9.5 & - & - & - & - & 15.6 & 23.8 & 10.2 & - & - & - \\
\hline SPM9-1 & 10.9 & - & - & - & - & - & 15.1 & 21.1 & 8.1 & - & - & - \\
\hline SPM3-7 & - & - & - & - & - & - & 16.8 & 26.2 & 10.2 & - & - & - \\
\hline SPM9-2 & 8.7 & - & - & - & - & - & 15.0 & 22.5 & 10.2 & - & - & - \\
\hline SPM3-6 & 10.0 & - & - & - & - & - & 17.3 & 22.0 & 12.4 & - & - & - \\
\hline SPM3-1 & 13.3 & - & - & - & - & - & 16.8 & 23.5 & 9.2 & - & - & - \\
\hline SRK1-2 & 17.6 & - & - & - & - & - & 17.9 & 23.6 & 8.7 & - & - & - \\
\hline SRK2-3 & 11.1 & - & - & - & - & - & 15.6 & 18.5 & 11.0 & - & - & - \\
\hline SRK1-1 & 9.6 & - & - & - & - & - & 13.8 & 20.4 & 8.7 & - & - & - \\
\hline SPM 3-5 & 9.2 & - & - & - & - & - & 15.7 & 23.0 & 10.7 & - & - & - \\
\hline \multicolumn{13}{|c|}{ Micromonospora } \\
\hline $\mathrm{CH} 7-4 \mathrm{~m}$ & - & - & 15.4 & - & - & - & - & 9.1 & 9.6 & - & - & - \\
\hline \multicolumn{13}{|l|}{ Streptomyces } \\
\hline LKB1-4 & - & - & - & - & - & - & 8.5 & - & - & - & - & - \\
\hline LKB1-1 & - & 9.5 & 9.6 & - & 14.0 & - & - & 10.2 & - & - & 13.3 & - \\
\hline PWB-011 & - & - & - & - & - & - & 9.5 & 13.6 & 9.7 & - & - & - \\
\hline BM2-4 & 16.3 & 20.8 & 12.0 & - & - & - & 19.5 & 21.4 & 12.1 & - & - & - \\
\hline CPB1-13 & 22.7 & 18.6 & 13.3 & - & - & - & 15.5 & 17.8 & 12.8 & - & - & - \\
\hline LKB1-6 & 9.0 & - & - & - & - & - & - & - & - & - & - & - \\
\hline LKB1-5 & - & 9.3 & 13.9 & - & - & - & - & - & - & - & - & - \\
\hline LKB1-7 & - & - & - & - & - & - & - & 9.3 & - & - & - & - \\
\hline PWB-010 & - & - & - & - & - & - & - & 8.1 & 15.0 & 11.9 & - & - \\
\hline PWB-016 & - & - & - & 9.3 & - & - & - & - & 12.3 & 10.2 & - & - \\
\hline
\end{tabular}

S, Staphylococcus aureus ATCC 25923; K, Kocuria rhizophila ATCC 9341; B, Bacillus subtilis ATCC 6633; E, Escherichia coli NIHJ KC213; C, Candida albicans KF1; M, Mucor racemosus IFO 4581. -, no activity. 
against tested microorganisms. The isolate $\mathrm{CH} 7-4 \mathrm{~m}$ identified as M. chersina ATCC $27029^{\mathrm{T}}$, exhibited activities against $S$. aureus ATCC 25923, K. rhizophila ATCC 9341, B. subtilis ATCC 6633. In addition, the isolate PWB-004 identified as Micromonospora olivasterospora, exhibited activities against B. subtilis ATCC 6633.

Ten Streptomyces isolates showed the antimicrobial activities against tested microorganisms. However, six isolates (SRN1-2, KK5-10, KRN1-1, CH3-1, SRN1-1 and LKB1-11) did not show any antimicrobial activities when cultured in four different media. Among the active isolates, anti-Gram-positive bacteria activity could be observed in most isolates, while antiGram-negative bacteria, anti-yeast, and anti-mold activities were observed only 5,8 , and 4 isolates, respectively. The detailed antimicrobial activities of the Streptomyces isolates are shown in Tables 2 and 3. No antimicrobial activities were observed for the members of the genus Nocardia and Verrucosispora.

According to the screening of their antimicrobial activities, the good example could be observed in the members of the Salinispora group (Tables 2 and 3). No activities were observed when they were grown in 301 medium, but almost all isolates showed the activities against tested Gram-positive bacteria when they were grown in 51 medium. Besides this, the difference between strains within the same species but different isolates are other factors for the determination of the antimicrobial activity. For example, isolate SRK1-2 identified as $S$. arenicola showed activities in 54 and 51 media. On the basis of this study, it suggested that the screening should be determined using various different media. Moreover, the same species of the isolates does not mean the same activities will be observed. In addition, the further elucidation of bioactive compounds of $S$. arenicola, Micromonospora and Streptomyces isolates mentioned should be done as previously reported (Abdelmohsen et al., 2014; Supong et al., 2012).

\section{CONCLUSION}

In this study, the marine actinomycetes, S. arenicola, Micromonospora aurantiaca, Micromonospora chalcea, Micromonospora chersina, Micromonospora marina, $M$. olivasterospora, Micromonospora tulbaghiae, V. gifhornensis, $V$. sediminis, Nocardia testacea, Streptomyces coelicoflavus, Streptomyces gulbargensis, Streptomyces olivaceoviridis, Streptomyces platensis, Streptomyces radiopugnans, Streptomyces rimosus, Streptomyces sanyensis, Streptomyces somaliensis, and Streptomyces tritolerans isolates were distributed in sand, mangrove sediments, marine sediments, and marine sponges collected from Chumphon, Chonburi, Phuket, Trang, and Krabi provinces in Thailand. Micromonospora, Salinispora, and Streptomyces isolates showed antimicrobial activity against $S$. aureus ATCC 25923, K. rhizophila ATCC 9341, B. subtilis ATCC 6633, E. coli NIHJ KC213, C. albicans KF1, and M. racemosus IFO 4581 .

\section{ACKNOWLEDGMENTS}

We thank Prof. Dr. Yoko Takahashi, Prof. Dr. Kazuro Shiomi, Dr. Atsuko Matsumoto, and the staffs of Kitasato Institute for Life Sciences, Kitasato University, Tokyo, Japan for supporting the screening and sequencing of the isolates.

\section{FINANCIAL SUPPORT AND SPONSORSHIP}

This study was supported by the Thailand Research Fund via a 2011 Royal Golden Jubilee Ph. D. Program as a scholarship to W. P. and we are grateful to the Plant Genetic Conservation Project under the Royal Initiative of Her Royal Highness Princess Maha Chakri Sirindhorn for the marine sediment samples collection.

\section{CONFLICT OF INTEREST}

There are no conflicts of interest.

\section{REFERENCES}

Abdelmohsen UR, Bayer K, Hentschel U. Diversity, abundance and natural products of marine sponge-associated actinomycetes. Nat Prod Rep, 2014; 31:381-99.

Berdy J. Bioactive microbial metabolites. J Antibiot, 2005; $58: 1-26$.

Bister B, Bischoff D, Ströbele M, Riedlinger J, Reicke A, Wolter F, Bull AT, Zähner H, Fiedler HP, Süssmuth RD. Abyssomicin C-A polycyclic antibiotic from a marine Verrucosispora strain as an inhibitor of the p-aminobenzoic acid/tetrahydrofolate biosynthesis pathway. Angew Chem Int Ed Engl, 2004; 43(19):2574-6.

Goodfellow M, Williams ST, Mordarski M. Actinomycetes in biotechnology. Academic Press Inc, London, UK, pp 1-88, 1988.

Inahashi Y, Matsumoto A, Omura S, Takahashi Y. Streptosporangium oxazolinicum $\mathrm{sp}$. nov., a novel endophytic actinomycete producing new antitrypanosomal antibiotics, spoxazomicins. J Antibiot (Tokyo), 2011; 64(4):297-302.

Jensen PR, Williams PG, Oh DC, Zeigler L, Fenical W. Speciesspecific secondary metabolite production in marine actinomycetes of the genus Salinispora. Appl Environ Microbiol, 2007; 73(4):1146-52.

Kageyama A, Yazawa K, Nishimura K, Mikami Y. Nocardia testaceus $\mathrm{sp}$. nov. and Nocardia senatus $\mathrm{sp}$. nov., isolated from patients in Japan. Microbiol Immunol, 2004; 48:271-6.

Kawamoto I. Genus Micromonospora. In: Williams ST, Sharpe ME, Holt JG (eds.). Bergey's manual of systematic bacteriology, vol. 4, Williams \& Wilkins, Baltimore, MD, pp 2442-50, 1989.

Kwon HC, Kauffman CA, Jensen PR, Fenical W. Marinomycins A-D, antitumor-antibiotics of a new structure class from a marine actinomycete of the recently discovered genus "Marinispora". J Am Chem Soc, $2006 ; 128(5): 1622-32$.

Lane DJ. 16S/23S rRNA sequencing. In Strackbrandt E, Goodfellow M (eds.). Nucleic acid techniques in bacterial systematics, Wiley, Chichester, UK, pp 115-48, 1991.

Maldonado LA, Fenical W, Jensen PR, Kauffman CA, Mincer TJ, Ward AC, Bull AT, Goodfellow M. Salinispora arenicola gen. nov., sp. nov. and Salinispora tropica sp. nov., obligate marine actinomycetes belonging to the family Micromonosporaceae. Int J Syst Evol Microbiol, 2005; 55:1759-66.

Mao J, Tang Q, Zhang Z, Wang W, Wei D, Huang Y, Liu Z, Shi Y, Goodfellow M. Streptomyces radiopugnans sp. nov., a radiation-resistant actinomycete isolated from radiation-polluted soil in China. Int J Syst Evol Microbiol, 2007; 57:2578-82.

Qin S, Chen H-H, Zhao G-Z, Li J, Zhu W-Y, Xu L-H, Jiang J-H, Li W-J. Abundant and diverse endophytic actinobacteria associated with medicinal plant Maytenus austro- yunnanensis in Xishuangbanna tropical rain forest revealed by culture-dependent and culture-independent methods. Envion Microbiol Report, 2012; 4:522-31.

Qin S1, Li J, Chen HH, Zhao GZ, Zhu WY, Jiang CL, Xu LH, Li WJ. Isolation, diversity, and antimicrobial activity of rare actinobacteria from medicinal plants of tropical rain forests in Xishuangbanna, China. Appl Environ Microbiol, 2009; 75(19):6176-86.

Rheims H, Schumann P, Rohde M, Stackebrandt E Verrucosispora gifhornensis gen. nov., sp. nov., a newmember of the 
actinobacterial family Micromonosporaceae. Int J Syst Bacteriol, 1998; 48:1119-27.

Shirling EB, Gottlieb D. Methods for characterization of Streptomyces species. Int J Syst Bacteriol, 1966; 16:313-40.

Sripreechasak P, Tanasupawat S, Matsumoto A, Inahashi Y, Suwanborirux K, Takahashi Y. Identification and antimicrobial activity of actinobacteria from soils in southern Thailand. Trop Biomed, 2013; 30(1):46-55.

Sripreechasak P, Suwanborirux K, Tanasupawat T. Characterization and antimicrobial activity of Streptomyces strains from soils in southern Thailand. J App Pharm Sci, 2014; 4(10):024-031.

Staneck JL, Roberts GD. Simplified approach to identification of aerobic actinomycetes by thin-layer chromatography. Appl Microbiol, $1974 ; 28: 226-31$.

Stackebrandt E, Rainey FA, Ward-Rainey NL. Proposal for a new hierarchic classification system, Actinobacteria classis nov. Int J Syst Bacteriol, 1997; 47:479491.

Suriyachadkun C, Chunhametha S, Thawai C, Tamura T, Potacharoen W, Kirtikara K, Sanglier JJ. Planotetraspora thailandica sp. nov., isolated from soil in Thailand. Int J Syst Evol Microbiol, 2009; 59:1632-7.
Supong K, Thawai C, Suwanborirux K, Choowong W, Supothina S, Pittayakhajonwut P. Antimalarial and antitubercular $C$-glycosylated benz[a]anthraquinones from the marine-derived Streptomyces $\mathrm{sp}$. BCC45596. Phytochem Lett, 2012; 5:651-6.

Tamaoka J. Determination of DNA base composition. In Goodfellow M, O'Donnel AG (eds.). Chemical methods in prokaryotic systematics, Wiley, Chichester, UK, pp 463-70, 1994.

Zhang H, Lee YK, Zhang W, Lee HK. Culturable actinobacteria from the marine sponge Hymeniacidon perleve: isolation and phylogenetic diversity by $16 \mathrm{~S}$ rRNA gene-RFLP analysis. Antonie Van Leeuwenhoek, 2006; 90(2):159-69.

How to cite this article:

Phongsopitanun W, Suwanborirux K, Tanasupawat S. Distribution and antimicrobial activity of Thai marine actinomycetes. J Appl Pharm Sci, 2019; 9(02):129-134. 